Paolo Foschi, Stefano Pagliarani and Andrea Pascucci

Black-Scholes Formulae for Asian Options in Local Volatility Models

Quaderni di Dipartimento

Serie Ricerche 2011, n. 7

ISSN 1973-9346

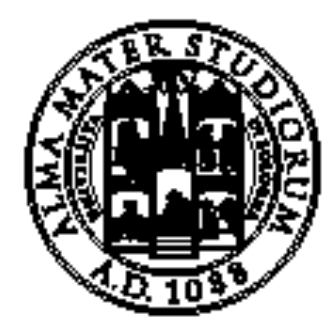

ALMA MATER STUDIORUM UNIVERSITA DI BOLOGNA

Dipartimento di Scienze Statistiche "Paolo Fortunati" 


\title{
Black-Scholes formulae for Asian options in local volatility models
}

\author{
Paolo Foschi, Stefano Pagliarani ${ }^{\dagger}$ And Andrea Pascucci ${ }^{\ddagger}$
}

July 29, 2011

\begin{abstract}
We develop approximate formulae expressed in terms of elementary functions for the density, the price and the Greeks of path dependent options of Asian style, in a general local volatility model. An algorithm for computing higher order approximations is provided. The proof is based on a heat kernel expansion method in the framework of hypoelliptic, not uniformly parabolic, partial differential equations.
\end{abstract}

\section{Introduction}

Asian options are path dependent derivatives whose payoff depends on some form of averaging prices of the underlying asset. Asian-style derivatives are widely traded in both exchanges and over-the-counter markets and constitute an important family of contracts with several applications.

From the theoretical point of view, arithmetically-averaged Asian options have attracted an increasing interest in the last decades due to the awkward nature of the related mathematical problems. Indeed, even in the standard Black \& Scholes (BS) model, when the underlying asset is a geometric Brownian motion, the distribution of the arithmetic average is not lognormal and it is quite complex to analytically characterize it. An integral representation was obtained in the pioneering work by Yor [46, 47], but with limited practical use in the valuation of Asian options.

Later on, Geman and Yor [21] gave an explicit representation of the Asian option prices in terms of the Laplace transform of hypergeometric functions. However, several authors (see Shaw [38], Fu, Madan and Wang

\footnotetext{
*Email: paolo.foschi2@unibo.it, Dipartimento di Scienze Statistiche "Paolo Fortunati", Università di Bologna, Via Belle Arti 41, 40126 Bologna, Italy

${ }^{\dagger}$ Email: stefanop@math.unipd.it, Dipartimento di Matematica, Università di Padova, Via Trieste 63, 35121 Padova, Italy

‡Email: pascucci@dm.unibo.it, Dipartimento di Matematica, Università di Bologna, Piazza di Porta S. Donato 5, 40126 Bologna, Italy
} 
[19], Dufresne [17]) noticed the greater difficulty of pricing Asian options with short maturities or small volatilities using the analytical method in [21]. This is also a disadvantage of the Laguerre expansion proposed by Dufresne [15]. In [39] Shaw used a contour integral approach based on Mellin transforms to improve the accuracy of the results in the case of low volatilities, albeit at a higher computational cost.

Several other numerical approaches to price efficiently Asian options in the BS model have been attempted. Monte Carlo simulation techniques were discussed by Kemna and Vorst [31], Boyle, Broadie and Glasserman [6], Fu, Madan and Wang [19], Jourdain and Sbai [29] and Guasoni and Robertson [24]. Takahashi and Yoshida [42] used Monte Carlo simulation combined with an asymptotic method based on Malliavin-Watanabe calculus. Linetsky [34] analyzed the problem using the spectral theory of singular Sturm-Liouville operators and obtained an eigenfunction expansion of the Asian option pricing function in the basis of Whittaker functions: Linetsky's series formula gives very accurate results, however it may converge slowly in the case of low volatility becoming computationally expensive.

Concerning the PDE approach, the averaging price for an Asian option is usually described by introducing an additional stochastic process (cf. Dewynne and Wilmott [13]): state augmentation converts the pathdependent problem into an equivalent path-independent and Markovian problem. Increasing the dimension causes the resulting pricing PDE to be degenerate and not uniformly parabolic: theoretical results for a class of hypoelliptic PDEs, which includes Asian equations of European and American style as particular cases, were proved by Barucci, Polidoro and Vespri [4], Di Francesco, Pascucci and Polidoro [14], Pascucci [35] and Bally and Kohatsu-Higa [3]. We recall that, in the BS model and for special homogeneous payoff functions, it is possible to reduce the study of Asian options to a PDE with only one state variable: PDE reduction techniques were initiated by Ingersoll [28] and developed by Rogers and Shi [37] and Zhang [48]. Similarly, Vecer [44], [45] used a change of numeraire technique to reduce the Asian pricing problem to a single spatial variable PDE that can be solved numerically by standard schemes; moreover, Glasgow and Taylor [22], Taylor [43] and Caister, O'Hara and Govinder [8] proposed a general study of symmetries for the Asian PDE and found other nontrivial reductions of the pricing equation.

The reduced PDE formulation was used by Dewynne and Shaw [12] to derive accurate approximation formulae for Asian-rate Call options in the BS model by a matched asymptotic expansion. In general, analytical approaches based on perturbation theory and asymptotic expansions have several advantages with respect to standard numerical methods: first of all, analytical approximations give closed-form solutions that exhibit an explicit dependency of the results on the underlying parameters. Moreover analytical approaches produce much better and much faster sensitivities than 
numerical methods, although often accurate error estimates are not trivial to obtain. Asymptotic methods for Asian options with explicit error bounds were studied by Corielli, Foschi and Pascucci [9] by PDEs techniques, and by Kunitomo and Takahashi [32], Shiraya and Takahashi [40], Shiraya, Takahashiand and Toda [41], Gobet [23] by Malliavin calculus techniques.

While most of the literature focuses on the log-normal dynamics and provides ad-hoc methods for pricing Asian options in the special case of the BS model, there are some notable exceptions given by the very recent papers by Bayraktar and Xing [5], Cai and Kou [7] where models with jumps are considered. Moreover Dufresne [16], Dassios and Nagaradjasarma [11] consider the square-root dynamics.

In this paper we consider the pricing problem for Asian options under a local volatility (LV), possibly time-dependent, model. In this general framework, dimension reduction is not possible anymore: then our idea is to use the natural geometric-differential structure of the pricing operator regarded as a hypoelliptic (not uniformly parabolic) PDE of Kolmogorov type in $\mathbb{R}^{3}$. Our main results are explicit, BS-type approximation formulae not only for the option price, but also for the the terminal distribution of the asset and the average; further we also get explicit approximation formulae for the Greeks that appear to be new also in the standard log-normal case. Under the BS dynamics, our formulae are extremely accurate if compared with other results in the literature. In a general LV model, we have only a comparison with Monte Carlo simulations and in this case the results are effectively exact under standard parameter regimes. An interesting feature of our methodology is that, in the case of linear payoff functions of the form

$$
\varphi(S, A)=\varphi_{1}+\varphi_{2} S+\varphi_{3} A,
$$

with $\varphi_{1}, \varphi_{2}, \varphi_{3} \in \mathbb{R}$, the resulting approximation formula is exact at order zero and all the higher order terms are null (cf. Remark 2.4): this seems a significant consistency result. Since the approximation formulae for a general LV model are rather long, in this paper we only give the explicit expression in the first order case and provide a general iterative algorithm for computing the higher order approximations, which can be easily implemented in any symbolic computational software: the Mathematica notebook containing the general formulae and the experiments reported in Section 3 is available in the web-site of the authors. The theoretical problem of the convergence and the error estimates for the expansion will not be addressed here: Corielli, Foschi and Pascucci [9] recently found global error bounds, based on Schauder estimates, for a similar expansion for degenerate PDEs of Asian type. It turns out that theoretical error estimates are generally very conservative and experimental results show that the explicit formulae have very good precision even in extreme cases; further, the assumptions needed to prove the theoretical results rule out models of practical interest such as the CEV 
model. Finally, we mention that our method can also be applied to other path-dependent models driven by hypoelliptic degenerate PDEs, namely the models proposed by Hobson and Rogers [26] and Foschi and Pascucci [18]. Moreover, jump-diffusion models can be considered as well. We aim to come back to these and other topics in a forthcoming paper.

The remainder of the paper is organized as follows. Section 2 describes arithmetic and geometric Asian options, sets up the valuation problem by PDE methods and introduces our notations. Subsection 2.1 presents the approximation methodology and Subsection 2.2 states some preliminary result on linear SDEs. Subsection 2.3 contains the main results of the paper and in Subsection 2.4 the first order approximation formulae are derived in the case of time-independent coefficients. Section 3 presents computational results.

\section{Asian options and linear SDEs}

We consider a standard market model where there is a risky asset $S$ following the stochastic differential equation

$$
d S_{t}=(r(t)-q(t)) S_{t} d t+\sigma\left(t, S_{t}\right) S_{t} d W_{t}
$$

under the risk-neutral measure. In (2.1), $r(t)$ and $q(t)$ denote the risk-free rate and the dividend yield at time $t$ respectively, $\sigma$ is the local volatility function and $W$ is a standard real Brownian motion.

The averaging prices for an Asian option are usually described by the additional state process

$$
d A_{t}=f\left(t, S_{t}\right) d t .
$$

In particular, for the continuously sampled Asian options we typically have

$$
\begin{array}{cl}
f(t, s)=g(t) s & \text { (arithmetic average option), } \\
f(t, s)=g(t) \log s & \text { (geometric average option), }
\end{array}
$$

where $g$ is some weight function. In the sequel, for simplicity, we shall only consider the case $g \equiv 1$ even if our methodology can include a generic positive weight $g$. By usual no-arbitrage arguments, the price of a European Asian option with payoff function $\varphi$ is given by

$$
V\left(t, S_{t}, A_{t}\right)=e^{-\int_{t}^{T} r(\tau) d \tau} u\left(t, S_{t}, A_{t}\right)
$$

where

$$
u\left(t, S_{t}, A_{t}\right)=E\left[\varphi\left(S_{T}, A_{T}\right) \mid S_{t}, A_{t}\right] .
$$


Typical payoff functions are given by

$$
\begin{array}{ll}
\varphi(S, A)=\left(\frac{A}{T}-K\right)^{+} & (\text {fixed strike arithmetic Call }), \\
\varphi(S, A)=\left(S-\frac{A}{T}\right)^{+} & (\text {floating strike arithmetic Call }), \\
\varphi(S, A)=\left(e^{\frac{A}{T}}-K\right)^{+} & (\text {fixed strike geometric Call }), \\
\varphi(S, A)=\left(S-e^{\frac{A}{T}}\right)^{+} & (\text {floating strike geometric Call }) .
\end{array}
$$

Clearly, Asian Puts can be considered as well: we recall that symmetry results, analogous to the standard Put-Call parity, between the floating and fixed-strike Asian options were proved by Henderson and Wojakowski [25].

By Feynman-Kac representation, the price function $u$ in (2.3) is the solution to the Cauchy problem

$$
\begin{cases}L u(t, s, a)=0, & t<T, s, a \in \mathbb{R}_{+}, \\ u(T, s, a)=\varphi(s, a), & s, a \in \mathbb{R}_{+},\end{cases}
$$

where $L$ is the ultra-parabolic ${ }^{1}$ pricing operator:

$$
L=\frac{\sigma^{2}(t, s) s^{2}}{2} \partial_{s s}+(r(t)-q(t)) s \partial_{s}+f(t, s) \partial_{a}+\partial_{t} .
$$

Under suitable regularity and growth conditions, existence and uniqueness of the solution to the Cauchy problem for $L$ were proved by Barucci, Polidoro and Vespri [4].

Remark 2.1. Consider the geometric Asian option under the BS dynamics: by the standard log-change of variable

$$
X_{t}=\left(\log S_{t}, A_{t}\right)
$$

equations (2.1)-(2.2) are transformed into the system of linear SDEs

$$
\begin{aligned}
& d X_{t}^{1}=\left(r(t)-q(t)-\frac{\sigma^{2}(t)}{2}\right) d t+\sigma(t) d W_{t}, \\
& d X_{t}^{2}=X_{t}^{1} d t
\end{aligned}
$$

Thus $X$ is a Gaussian process with 2-dimensional normal transition density $\Gamma$ that is the fundamental solution of the differential operator

$$
K:=\frac{\sigma^{2}(t)}{2}\left(\partial_{x_{1} x_{1}}-\partial_{x_{1}}\right)+(r(t)-q(t)) \partial_{x_{1}}+x_{1} \partial_{x_{2}}+\partial_{t}, \quad x \in \mathbb{R}^{2} .
$$

\footnotetext{
${ }^{1} L$ is defined on $\mathbb{R}^{3}$ but contains only the second order derivative w.r.t the variable $s$ : thus $L$ is not a uniformly parabolic operator.
} 
The expression of $\Gamma$ is given in Subsection 2.2 and explicit formulae for fixed and floating strike geometric Asian options can be easily found: Kemna and Vorst [31] have derived the first exact valuation formula for the geometric average Asian option. We also mention Angus [2] who considered more general payoffs.

\subsection{Approximation methodology}

In this subsection we briefly recall the approximation technique proposed by Pagliarani and Pascucci in [36] and adapt it to Asian options. This allows us to get a new expansion formula for the fundamental solution of the arithmetic Asian operator (cf. (2.4) with $f(t, s)=s$ ). The coefficients of the expansion will be computed explicitly in Subsection 2.3.

We consider the operator

$$
L=\frac{\alpha(t, s)}{2} \partial_{s s}+(r(t)-q(t)) s \partial_{s}+s \partial_{a}+\partial_{t}
$$

where

$$
\alpha(t, s)=\sigma^{2}(t, s) s^{2}
$$

We assume that $\alpha$ is a suitably smooth, positive function and we take the Taylor expansion of $\alpha(t, \cdot)$ about $s_{0} \in \mathbb{R}_{+}$: then formally we get

$$
L=L^{0}+\sum_{k=1}^{\infty}\left(s-s_{0}\right)^{k} \alpha_{k}(t) \partial_{s s}
$$

where, setting $\alpha_{0}(t)=\alpha\left(t, s_{0}\right)$,

$$
L^{0}=\frac{\alpha_{0}(t)}{2} \partial_{s s}+(r(t)-q(t)) s \partial_{s}+s \partial_{a}+\partial_{t},
$$

is the leading term in the approximation of $L$ and

$$
\alpha_{k}(t)=\frac{1}{2 k !} \partial_{s}^{k} \alpha\left(t, s_{0}\right), \quad k \geq 1 .
$$

Notice that $L^{0}$ in $(2.9)$ is the Kolmogorov operator associated to the system

$$
\begin{aligned}
d S_{t} & =(r(t)-q(t)) S_{t} d t+\sqrt{\alpha_{0}(t)} d W_{t}, \\
d A_{t} & =S_{t} d t .
\end{aligned}
$$

Remark 2.2. As in the geometric case, (2.10) is a system of linear SDEs whose solution $(S, A)$ has a 2-dimensional normal transition density $\Gamma^{0}$. Moreover $\Gamma^{0}$ is the Gaussian fundamental solution of $L^{0}$ in (2.9) and its explicit expression will be given in Subsection 2.2. 
Following [36], the fundamental solution $\Gamma$ of the pricing operator $L$ in (2.6) admits an expansion of the form

$$
\Gamma(t, s, a ; T, S, A)=\sum_{n=0}^{\infty} G^{n}(t, s, a ; T, S, A)
$$

where

$$
G^{0}(t, s, a ; T, S ; A)=\Gamma^{0}(t, s, a ; T, S ; A), \quad t<T, s, a, S, A \in \mathbb{R},
$$

and $G^{n}(\cdot ; T, S, A)$, for any $n \geq 1$ and $T, S, A$, is defined recursively in terms of the following sequence of Cauchy problems posed on $]-\infty, T\left[\times \mathbb{R}^{2}\right.$ :

$$
\left\{\begin{array}{l}
L^{0} G^{n}(t, s, a ; T, S ; A)=-\sum_{k=1}^{n}\left(s-s_{0}\right)^{k} \alpha_{k}(t) \partial_{s s} G^{n-k}(t, s, a ; T, S ; A), \\
G^{n}(T, s, a ; T, S ; A)=0, \quad s, a \in \mathbb{R} .
\end{array}\right.
$$

For instance, $G^{1}(\cdot ; T, S, A)$ is defined by

$$
\left\{\begin{array}{l}
L^{0} G^{1}(t, s, a ; T, S ; A)=-\left(s-s_{0}\right) \alpha_{1}(t) \partial_{s s} G^{0}(t, s, a ; T, S ; A), \\
G^{1}(T, s, a ; T, S ; A)=0, \quad s, a \in \mathbb{R},
\end{array}\right.
$$

and for $n=2$ we have

$$
\left\{\begin{aligned}
& L^{0} G^{2}(t, s, a ; T, S ; A)=-\left(s-s_{0}\right) \alpha_{1}(t) \partial_{s s} G^{1}(t, s, a ; T, S ; A) \\
& \quad-\left(s-s_{0}\right)^{2} \alpha_{2}(t) \partial_{s s} G^{0}(t, s, a ; T, S ; A), \\
& G^{2}(T, s, a ; T, S ; A)=0, \quad s, a \in \mathbb{R} .
\end{aligned}\right.
$$

Remark 2.3. Under the BS dynamics, the diffusion coefficient in (2.7) is of the form

$$
\alpha(t, s)=\sigma(t) s^{2}
$$

where $t \mapsto \sigma(t)$ is a deterministic function. Thus

$$
\alpha_{n} \equiv 0, \quad n \geq 3,
$$

and in this particular case, the sequence of Cauchy problems in (2.12) reduces to

$$
\left\{\begin{aligned}
& L^{0} G^{n}(t, s, a ; T, S ; A)=-\left(s-s_{0}\right) \alpha_{1}(t) \partial_{s s} G^{n-1}(t, s, a ; T, S ; A) \\
&-\left(s-s_{0}\right)^{2} \alpha_{2}(t) \partial_{s s} G^{n-2}(t, s, a ; T, S ; A), \\
& G^{n}(T, s, a ; T, S ; A)=0, \quad s, a \in \mathbb{R}
\end{aligned}\right.
$$

for $n \geq 2$. A similar reduction holds for any diffusion coefficient of polynomial type in the variable $s$. 
In general, the sequence $\left(G^{n}\right)_{n \geq 1}$ defined by (2.12) can be computed explicitly by an iterative algorithm: this will be detailed in Subsection 2.3 by using the results on linear SDEs presented in Subsection 2.2. In particular, it turns out that

$$
G^{n}(t, s, a ; T, S ; A)=J_{t, T, s, a}^{n} G^{0}(t, s, a ; T, S ; A), \quad n \geq 0,
$$

where $J_{t, T, s, a}^{0}$ is the identity operator and, for $n \geq 1, J_{t, T, s, a}^{n}$ is a differential operator, acting in the variables $s, a$, of the form

$$
J_{t, T, s, a}^{n}=\sum_{k=0}^{n} s^{k} \sum_{i=2}^{3 n} \sum_{j=0}^{i} f_{i-j, j, k}^{n}(t, T) \frac{\partial^{i}}{\partial s^{i-j} \partial a^{j}},
$$

and the coefficients $f_{i-j, j, k}^{n}$ are deterministic functions whose explicit expression can be computed iteratively as in Theorem 2.7 and Remark 2.8 below. Thus, by (2.11), the $N$-th order approximation of $\Gamma$ is given by

$$
\Gamma(t, s, a ; T, S, A) \approx \sum_{n=0}^{N} J_{t, T, s, a}^{n} G^{0}(t, s, a ; T, S, A) .
$$

Moreover we have the following $N$-th order approximation formula for the price of an arithmetic Asian option with payoff function $\varphi$ :

$$
\begin{aligned}
u\left(t, S_{t}, A_{t}\right) & =\iint \Gamma(t, s, a ; T, S, A) \varphi(S, A) d S d A \\
& \approx \iint \Gamma^{N}(t, s, a ; T, S, A) \varphi(S, A) d S d A \\
& =\sum_{n=0}^{N} J_{t, T, s, a}^{n} C_{0}(t, s, a)=: u_{N}\left(t, S_{t}, A_{t}\right)
\end{aligned}
$$

where

$$
C_{0}(t, s, a)=\iint \Gamma^{0}(t, s, a ; T, S, A) \varphi(S, A) d S d A .
$$

Notice that $C_{0}$ is the price of a geometric Asian option under the BS dynamics and therefore for typical payoff functions it has a closed form expression. Similarly we obtain explicit approximation formulae for the Greeks and for any other payoff which admits an explicit pricing formula in the geometric case.

Remark 2.4. Let us consider an affine payoff function of the form

$$
\varphi(S, A)=\varphi_{1}+\varphi_{2} S+\varphi_{3} A,
$$

with $\varphi_{1}, \varphi_{2}, \varphi_{3} \in \mathbb{R}$. Then a direct computation shows that

$$
C_{0}(t, s, a)=\varphi_{1}+\left\langle\left(\varphi_{2}, \varphi_{3}\right), m_{t, s, a}(T)\right\rangle
$$


with $m_{t, s, a}(T)$ as in (2.30). Since $C_{0}(t, s, a)$ is again an affine function of $(s, a)$, we have that

$$
J_{t, T, s, a}^{n} C_{0}(t, s, a)=0, \quad \forall n \geq 1,
$$

and therefore $u_{N} \equiv u_{0}$ in (2.15), for any $N \in \mathbb{N}$. Moreover, by the uniqueness of the solution of the Cauchy problem for $L$, we also have $u_{0}=u$, that is when the payoff is an affine function of $S$ and $A$, then the first approximation is exact and all the higher order terms are null. Roughly speaking, this property follows from the fact that the differential operators $L$ and $L^{0}$ have the same first order part and only differ in the coefficient of their second order derivative.

\subsection{Non-degeneracy conditions for linear SDEs}

In this subsection we collect some preliminary results on linear SDEs that will be used in the derivation of the approximation formulae for the arithmetic density. First notice that equations (2.5) and (2.10) belong to the general class of linear SDEs

$$
d X_{t}=\left(B(t) X_{t}+b(t)\right) d t+\sigma(t) d W_{t}
$$

where $b, B$ and $\sigma$ are $L_{\text {loc }}^{\infty}$-functions with values in the space of $(N \times 1),(N \times$ $N)$ and $(N \times d)$-dimensional matrices respectively and $W$ is a $d$-dimensional uncorrelated Brownian motion, with $d \leq N$. The solution $X=X^{t, x}$ to (2.17) with initial condition $x \in \mathbb{R}^{N}$ at time $t$, is given explicitly by

$$
X_{T}=\Phi(t, T)\left(x+\int_{t}^{T} \Phi^{-1}(t, \tau) b(\tau) d \tau+\int_{t}^{T} \Phi^{-1}(t, \tau) \sigma(\tau) d W_{\tau}\right),
$$

where $T \mapsto \Phi(t, T)$ is the matrix-valued solution to the deterministic Cauchy problem

$$
\left\{\begin{array}{l}
\frac{d}{d T} \Phi(t, T)=B(T) \Phi(t, T), \\
\Phi(t, t)=I_{N} .
\end{array}\right.
$$

Moreover $X^{t, x}$ is a Gaussian process with expectation

$$
m_{t, x}(T):=E\left[X_{T}^{t, x}\right]=\Phi(t, T) R(x, t, T),
$$

where

$$
R(x, t, T)=x+\int_{t}^{T} \Phi^{-1}(t, \tau) b(\tau) d \tau
$$

and covariance matrix

$$
C(t, T)=\operatorname{cov}\left(X_{T}^{t, x}\right)=\Phi(t, T) M(t, T) \Phi(t, T)^{*},
$$


where

$$
M(t, T)=\int_{t}^{T} \Phi^{-1}(t, \tau) \sigma(\tau)\left(\Phi^{-1}(t, \tau) \sigma(\tau)\right)^{*} d \tau .
$$

The Kolmogorov operator associated with $X$ is

$$
\begin{aligned}
K & =\frac{1}{2} \sum_{i, j=1}^{N} c_{i j}(t) \partial_{x_{i} x_{j}}+\langle b(t)+B(t) x, \nabla\rangle+\partial_{t} \\
& =\frac{1}{2} \sum_{i, j=1}^{N} c_{i j}(t) \partial_{x_{i} x_{j}}+\sum_{i=1}^{N} b_{i}(t) \partial_{x_{i}}+\sum_{i, j=1}^{N} B_{i j}(t) x_{i} \partial_{x_{j}}+\partial_{t},
\end{aligned}
$$

where $\left(c_{i j}\right)=\sigma \sigma^{*}$.

Now we assume the following crucial condition:

[H.1] the matrix $C(t, T)$ (or equivalently, the matrix $M(t, T)$ ) is positive definite for any $T>t$.

Under this condition, $X_{T}^{t, x}$ has a transition density given by

$$
\Gamma_{K}(t, x, T, y)=\frac{1}{\sqrt{(2 \pi)^{N} \operatorname{det} C(t, T)}} e^{-\frac{1}{2}\left\langle C^{-1}(t, T)\left(y-m_{t, x}(T)\right), y-m_{t, x}(T)\right\rangle} .
$$

$\Gamma_{K}$ is also the fundamental solution of $K$ in (2.22). Condition [H.1] can be expressed in geometric-differential terms: in fact, it is known that [H.1] is equivalent to the following condition due to Hörmander [27]

$$
\operatorname{rank} \mathcal{L}\left(Y_{1}, \cdots, Y_{d}, Y\right)(t, x)=N+1, \quad(t, x) \in \mathbb{R}^{N+1},
$$

where $\mathcal{L}\left(Y_{1}, \cdots, Y_{d}, Y\right)$ denotes the Lie algebra generated by the vector fields in $\mathbb{R}^{N+1}$

$$
Y_{i}=\sum_{j=1}^{N} \sigma_{j i} \partial_{x_{j}}, \quad i=1, \ldots, d
$$

and

$$
Y=\langle B(t) x+b(t), \nabla\rangle+\partial_{t} .
$$

In other terms, $\mathcal{L}\left(Y_{1}, \cdots, Y_{d}, Y\right)(t, x)$ is the vector space spanned by the vector fields $Y_{1}, \cdots, Y_{d}, Y$, by their first order commutators $\left[Y_{k}, Y\right], k=1, \ldots, d$, where $\left[Y_{k}, Y\right] u:=Y_{k} Y u-Y Y_{k} u$ and by their higher order commutators $\left[Y_{j}, \ldots,\left[Y_{k}, Y\right] \ldots\right]$, evaluated at the point $(t, x)$.

Hörmander's condition and [H.1] are also equivalent to another condition from control theory: for any $T>0$, a curve $x:[0, T] \longmapsto \mathbb{R}^{N}$ is called $K$ admissible if it is absolutely continuous and satisfies

$$
x^{\prime}(t)=B(t) x(t)+b(t)+\sigma(t) w(t), \quad \text { a.e. in }[0, T],
$$


for a suitable function $w$ with values in $\mathbb{R}^{d}$ (notice the close analogy with the SDE (2.17)). The function $w$ is called the control of the path $x$. A fundamental result by Kalman, Ho and Narendra [30] states that [H.1] is equivalent to the following condition:

[H.3] for every $x_{0}, x_{1} \in \mathbb{R}^{N}$ and $T>0$, there exists a $K$-admissible path such that $x(0)=x_{0}$ and $x(T)=x_{1}$.

When $B$ and $\sigma$ are constant matrices, then [H.3] is equivalent to the well known Kalman's rank condition (we also refer to LaSalle [33] where this result first appeared)

$$
\operatorname{rank}\left(\begin{array}{llll}
\sigma & B \sigma & \ldots & B^{N-1} \sigma
\end{array}\right)=N .
$$

An analogous condition for time-dependent matrices $\sigma(t)$ and $B(t)$ was given by Coron [10], Agrachev and Sachkov [1].

The following simple result will be crucial in the sequel.

Proposition 2.5. Under assumption [H.1], we have

$$
\begin{aligned}
\nabla_{y} \Gamma_{K}(t, x, T, y) & =-\left(\Phi^{-1}(t, T)\right)^{*} \nabla_{x} \Gamma_{K}(t, x, T, y) \\
y \Gamma_{K}(t, x, T, y) & =\Phi(t, T)\left(R(x, t, T)+M(t, T) \nabla_{x}\right) \Gamma_{K}(t, x, T, y),
\end{aligned}
$$

for any $x, y \in \mathbb{R}^{N}$ and $t<T$.

Proof. The density $\Gamma_{K}$ in (2.23) can be rewritten in the equivalent form

$$
\Gamma_{K}(t, x, T, y)=\frac{e^{-\frac{1}{2}\left\langle M^{-1}(t, T)\left(\Phi^{-1}(t, T) y-R(x, t, T)\right), \Phi^{-1}(t, T) y-R(x, t, T)\right\rangle}}{\sqrt{(2 \pi)^{N} \operatorname{det} C(t, T)}} .
$$

By differentiating, we get

$$
\frac{\nabla_{x} \Gamma_{K}(t, x, T, y)}{\Gamma_{K}(t, x, T, y)}=M^{-1}(t, T)\left(\Phi^{-1}(t, T) y-R(x, t, T)\right)
$$

and

$$
\frac{\nabla_{y} \Gamma_{K}(t, x, T, y)}{\Gamma_{K}(t, x, T, y)}=-\left(\Phi^{-1}(t, T)\right)^{*} M^{-1}(t, T)\left(\Phi^{-1}(t, T) y-R(x, t, T)\right)=
$$

(by $(2.28)$ )

$$
=-\left(\Phi^{-1}(t, T)\right)^{*} \frac{\nabla_{x} \Gamma_{K}(t, x, T, y)}{\Gamma_{K}(t, x, T, y)},
$$

and this proves (2.26). Formula (2.27) follows immediately from (2.28). 


\subsection{Approximation formulae for the density}

We consider the operator

$$
L^{0}=\frac{\alpha_{0}(t)}{2} \partial_{s s}+\mu(t) s \partial_{s}+s \partial_{a}+\partial_{t}, \quad(t, s, a) \in \mathbb{R}^{3},
$$

that is the leading term in the approximation of arithmetic Asian options, as in (2.9) with

$$
\mu=r-q .
$$

According to notations of Subsection 2.2, we have $b=0$ and

$$
B(t)=\left(\begin{array}{cc}
\mu(t) & 0 \\
1 & 0
\end{array}\right) .
$$

Hence we have

$$
\begin{aligned}
\Phi(t, T)= & \left(\begin{array}{cc}
e^{\int_{t}^{T} \mu(\tau) d \tau} & 0 \\
\int_{t}^{T} e^{\int_{t}^{\tau_{1}} \mu\left(\tau_{2}\right) d \tau_{2}} d \tau_{1} & 1
\end{array}\right) \\
m_{t, s, a}(T)= & \left(\begin{array}{c}
s e^{\int_{t}^{T} \mu(\tau) d \tau} \\
a+s \int_{t}^{T} e^{\int_{t}^{\tau_{1}} \mu\left(\tau_{2}\right) d \tau_{2}} d \tau_{1}
\end{array}\right) \\
M(t, T)= & \int_{t}^{T} \alpha_{0}(\tau) e^{-2 \int_{t}^{\tau} \mu\left(\tau_{1}\right) d \tau_{1}} \\
& \cdot\left(\begin{array}{cc}
1 & -\int_{t}^{\tau} e^{\int_{t}^{\tau_{1}} \mu\left(\tau_{2}\right) d \tau_{2}} d \tau_{1} \\
-\int_{t}^{\tau} e^{\int_{t}^{\tau_{1}} \mu\left(\tau_{2}\right) d \tau_{2}} d \tau_{1} & \left(\int_{t}^{\tau} e^{\int_{t}^{\tau_{1}} \mu\left(\tau_{2}\right) d \tau_{2}} d \tau_{1}\right)^{2}
\end{array}\right) d \tau
\end{aligned}
$$

It is easy to verify that $M(t, T)$ (and the covariance matrix $C(t, T)$ ) is positive definite by checking Hörmander's condition [H.2]: indeed, the commutator of the vector fields

$$
Y_{1}=\sqrt{\alpha_{0}(t)} \partial_{s}, \quad Y=\mu(t) s \partial_{s}+s \partial_{a}+\partial_{t}
$$

is equal to

$$
\left[Y_{1}, Y\right]=\sqrt{\alpha_{0}(t)}\left(\mu(t) \partial_{s}+\partial_{a}\right)
$$

and therefore, assuming that $\alpha_{0}>0$, then the rank of the Lie algebra generated by $Y_{1}$ and $Y$ is equal to three.

If $\mu$ and $\alpha_{0}$ are constant, all computations can be carried out more explicitly and we have

$$
\begin{aligned}
& \Phi(t, T)=e^{(T-t) B}=\left(\begin{array}{cc}
e^{\mu(T-t)} & 0 \\
\frac{e^{\mu(T-t)}-1}{\mu} & 1
\end{array}\right) \\
& m_{t, s, a}(T)=\left(\begin{array}{c}
e^{\mu(T-t)} s \\
a+\frac{\left(e^{\mu(T-t)}-1\right) s}{\mu}
\end{array}\right) \\
& M(t, T)=\alpha_{0}\left(\begin{array}{cc}
\frac{1-e^{-2 \mu(T-t)}}{2 \mu} & -\frac{\left(1-e^{-\mu(T-t)}\right)^{2}}{2 \mu^{2}} \\
-\frac{\left(1-e^{-\mu(T-t)}\right)^{2}}{2 \mu^{2}} & \frac{4 e^{-\mu(T-t)}-e^{-2 \mu(T-t)}+2 \mu(T-t)-3}{2 \mu^{3}}
\end{array}\right) .
\end{aligned}
$$


In particular, for $\mu=0$ we get

$$
\Phi(t, T)=\left(\begin{array}{cc}
1 & 0 \\
T-t & 1
\end{array}\right), \quad M(t, T)=\alpha_{0}\left(\begin{array}{cc}
T-t & -\frac{(T-t)^{2}}{2} \\
-\frac{(T-t)^{2}}{2} & \frac{(T-t)^{3}}{3}
\end{array}\right) .
$$

Now let us recall the notation $\Gamma^{0}(t, s, a ; T, S, A)$ for the fundamental solution of $L^{0}$ in (2.29). In Corollary 2.6 below we reformulate more explicitly the properties of $\Gamma_{0}$ stated in Proposition 2.5. To this end and to shorten notations, we introduce the operator

$$
V_{t, T, s, a}=\left(\Phi(t, T)\left(\left(\begin{array}{c}
s \\
a
\end{array}\right)+M(t, T) \nabla_{s, a}\right)\right)_{1}
$$

where in general, for a given vector $Z$, we use the subscript $Z_{1}$ to denote its first component. Moreover, we define the differential operator $W_{t, T}^{i, j}$ as the composition

$$
W_{t, T, s, a}^{i, j}=W_{1, t, T, s, a}^{i} W_{2, t, T, s, a}^{j}
$$

of the first order operators

$$
W_{k, t, T, s, a}=\left(\left(\Phi^{-1}(t, T)\right)^{*} \nabla_{s, a}\right)_{k} \quad k=1,2 .
$$

As a direct application of Proposition 2.5, we have the following results which shows how the product and the derivatives with respect to the second set of variables of $\Gamma^{0}(t, s, a ; T, S, A)$ can be expressed in terms of the operators $V$ in (2.32) and $W$ and (2.33), acting in the first set of variables (i.e. $t, s, a)$.

Corollary 2.6. For any $t<T, s, a, S, A \in \mathbb{R}$ and $i, j \in \mathbb{N} \cup\{0\}$ we have

$$
\begin{aligned}
& S^{i} \Gamma^{0}(t, s, a ; T, S, A)=V_{t, T, s, a}^{i} \Gamma^{0}(t, s, a ; T, S, A), \\
& \frac{\partial^{i+j}}{\partial S^{i} \partial A^{j}} \Gamma^{0}(t, s, a ; T, S, A)=(-1)^{i+j} W_{t, T, s, a}^{i, j} \Gamma^{0}(t, s, a ; T, S, A) .
\end{aligned}
$$

Next we prove our main result.

Theorem 2.7. For any $n \geq 0$, the solution $G^{n}$ of problem (2.12) is given by

$$
G^{n}(t, s, a ; T, S, A)=J_{t, T, s, a}^{n} \Gamma^{0}(t, s, a ; T, S, A)
$$

where $\Gamma^{0}$ is the fundamental solution of $L^{0}$ in (2.29), $J_{t, T, s, a}^{0}$ is the identity operator and, for $n \geq 1, J_{t, T, s, a}^{n}$ is a differential operator of the form

$$
J_{t, T, s, a}^{n}=\sum_{k=0}^{n} s^{k} \sum_{i=2}^{3 n} \sum_{j=0}^{i} f_{i-j, j, k}^{n}(t, T) \frac{\partial^{i}}{\partial s^{i-j} \partial a^{j}} .
$$


The coefficients $f_{i-j, j, k}^{n}(t, T)$ in (2.38) are deterministic functions that can be determined iteratively by using the following alternative expression of $J^{n}$, $n \geq 1$, given in terms of the operators $V$ and $W$ in (2.32)-(2.33):

$$
J_{t, T, s, a}^{n}=\sum_{i=1}^{n} \int_{t}^{T} \alpha_{i}(\tau)\left(V_{t, \tau, s, a}-s_{0}\right)^{i} W_{t, \tau, s, a}^{2,0} \hat{J}_{t, \tau, T, s, a}^{n-i} d \tau
$$

where $\hat{J}_{t, \tau, T, s, a}^{0}$ is the identity operator and

$$
\hat{J}_{t, \tau, T, s, a}^{n}=\sum_{k=0}^{n} \sum_{i=2}^{3 n} \sum_{j=0}^{i} f_{i-j, j, k}^{n}(t, T) V_{t, \tau, s, a}^{k} W_{t, \tau, s, a}^{i-j, j} .
$$

Proof. We first remark that, if we assume $J_{t, T, s, a}^{n}$ and $\hat{J}_{t, \tau, T, s, a}^{n}$ as in (2.38) and (2.40) respectively, then by Corollary 2.6, for any $\tau \in] t, T[$, we have

$$
\begin{aligned}
& \int_{\mathbb{R}^{2}} \Gamma^{0}(t, s, a ; \tau, \xi, \eta) J_{\tau, T, \xi, \eta}^{n} \Gamma^{0}(\tau, \xi, \eta ; T, S, A) d \xi d \eta \\
& =\int_{\mathbb{R}^{2}} \hat{J}_{t, \tau, T, s, a}^{n} \Gamma^{0}(t, s, a ; \tau, \xi, \eta) \Gamma^{0}(\tau, \xi, \eta ; T, S, A) d \xi d \eta=
\end{aligned}
$$

(here $\hat{J}_{t, \tau, T, s, a}^{n}$ plays the role of the "adjoint" operator of $J_{\tau, T, \xi, \eta}^{n}$ )

$$
=\hat{J}_{t, \tau, T, s, a}^{n} \int_{\mathbb{R}^{2}} \Gamma^{0}(t, s, a ; \tau, \xi, \eta) \Gamma^{0}(\tau, \xi, \eta ; T, S, A) d \xi d \eta=
$$

(by the semigroup property of $\Gamma^{0}$ )

$$
=\hat{J}_{t, \tau, T, s, a}^{n} \Gamma^{0}(t, s, a ; T, S, A) .
$$

Next we prove the thesis by induction. For $n=1$, by the representation formula for the non-homogeneous parabolic Cauchy problem (2.13) with null final condition, we have

$G^{1}(t, s, a ; T, S, A)=$

$$
=\int_{t}^{T} \int_{\mathbb{R}^{2}} \Gamma^{0}(t, s, a ; \tau, \xi, \eta) \alpha_{1}(\tau)\left(\xi-s_{0}\right) \partial_{\xi \xi} \Gamma^{0}(\tau, \xi, \eta ; T, S, A) d \xi d \eta d \tau=
$$

(by $(2.35))$

$$
=\int_{t}^{T} \alpha_{1}(\tau)\left(V_{t, \tau, s, a}-s_{0}\right) \int_{\mathbb{R}^{2}} \Gamma^{0}(t, s, a ; \tau, \xi, \eta) \partial_{\xi \xi} \Gamma^{0}(\tau, \xi, \eta ; T, S, A) d \xi d \eta d \tau=
$$

(by parts and by (2.36))

$$
=\int_{t}^{T} \alpha_{1}(\tau)\left(V_{t, \tau, s, a}-s_{0}\right) W_{t, \tau, s, a}^{2,0} \int_{\mathbb{R}^{2}} \Gamma^{0}(t, s, a ; \tau, \xi, \eta) \Gamma^{0}(\tau, \xi, \eta ; T, S, A) d \xi d \eta d \tau=
$$


(by the semigroup property of $\Gamma^{0}$ )

$$
=\int_{t}^{T} \alpha_{1}(\tau)\left(V_{t, \tau, s, a}-s_{0}\right) W_{t, \tau, s, a}^{2,0} \Gamma^{0}(t, s, a ; T, S, A) d \tau .
$$

This proves (2.37)-(2.39) for $n=1$, that is

$$
G^{1}(t, s, a ; T, S, A)=J_{t, T, s, a}^{1} \Gamma^{0}(t, s, a ; T, S, A),
$$

where

$$
J_{t, T, s, a}^{1}=\int_{t}^{T} \alpha_{1}(\tau)\left(V_{t, \tau, s, a}-s_{0}\right) W_{t, \tau, s, a}^{2,0} d \tau .
$$

Using (2.42) and the explicit expression of the operators $V, W$ in (2.32)(2.33)-(2.34) given in terms of $\Phi, M$ in (2.30), we can easily rewrite $J_{t, T, s, a}^{1}$ in the form (2.38): we refer to Remark 2.8 below for the details and the derivation of the explicit expression of the coefficients $f_{i-j, j, k}^{1}$.

Now we assume that (2.37), (2.38) and (2.39) are valid for a generic but fixed $n$ and we prove them for $n+1$. Using again the standard representation formula for non-homogeneous parabolic Cauchy problem (2.12) with null final condition, we have

$$
G^{n+1}(t, s, a ; T, S, A)=\sum_{i=1}^{n+1} \int_{t}^{T} \alpha_{i}(\tau) I_{i}(t, s, y, \tau, T, S, A) d \tau,
$$

where

$$
\begin{aligned}
& I_{i}(t, s, y, \tau, T, S, A)= \\
& \int_{\mathbb{R}^{2}} \Gamma^{0}(t, s, a ; \tau, \xi, \eta)\left(\xi-s_{0}\right)^{i} \partial_{\xi \xi} G^{n+1-i}(\tau, \xi, \eta ; T, S, A) d \xi d \eta=
\end{aligned}
$$

(by Corollary 2.6)

$$
=\left(V_{t, \tau, s, a}-s_{0}\right)^{i} W_{t, \tau, s, a}^{2,0} \int_{\mathbb{R}^{2}} \Gamma^{0}(t, s, a ; \tau, \xi, \eta) G^{n+1-i}(\tau, \xi, \eta ; T, S, A) d \xi d \eta=
$$

(by the inductive hypothesis)

$$
=\left(V_{t, \tau, s, a}-s_{0}\right)^{i} W_{t, \tau, s, a}^{2,0} \int_{\mathbb{R}^{2}} \Gamma^{0}(t, s, a ; \tau, \xi, \eta) J_{\tau, T, \xi, \eta}^{n+1-i} \Gamma^{0}(\tau, \xi, \eta ; T, S, A) d \xi d \eta
$$

(by $(2.41)$ )

$$
=\left(V_{t, \tau, s, a}-s_{0}\right)^{i} W_{t, \tau, s, a}^{2,0} \hat{J}_{t, \tau, T, s, a}^{n+1-i} \Gamma^{0}(t, s, a ; T, S, A) .
$$

Plugging (2.44) into (2.43), we obtain formulae (2.37)-(2.39) and this concludes the proof. 
Remark 2.8. Starting from formula (2.42)

$$
J_{t, T, s, a}^{1}=\int_{t}^{T} \alpha_{1}(\tau)\left(V_{t, \tau, s, a}-s_{0}\right) W_{t, \tau, s, a}^{2,0} d \tau,
$$

we find the more explicit representation of $J_{t, T, s, a}^{1}$ in the form (2.38), that is

$$
J_{t, T, s, a}^{1}=\sum_{k=0}^{1} s^{k} \sum_{i=2}^{3} \sum_{j=0}^{i} f_{i-j, j, k}^{1}(t, T) \frac{\partial^{i}}{\partial s^{i-j} \partial a^{j}} .
$$

We first remark that, by the definition (2.32), (2.33) and (2.34) of the operators $V$ and $W$, we have

$$
\begin{aligned}
& V_{t, \tau, s, a}=s \Phi_{11}(t, \tau)+M_{11}(t, \tau) \Phi_{11}(t, \tau) \partial_{s}+M_{21}(t, \tau) \Phi_{11}(t, \tau) \partial_{a} \\
& W_{t, \tau, s, a}^{2,0}=\frac{1}{\Phi_{11}(t, \tau)^{2}} \partial_{s s}-\frac{2 \Phi_{21}(t, \tau)}{\Phi_{11}(t, \tau)^{2}} \partial_{s a}+\frac{\Phi_{21}(t, \tau)^{2}}{\Phi_{11}(t, \tau)^{2}} \partial_{a a},
\end{aligned}
$$

where $\Phi_{i j}$ and $M_{i j}$ denote the components of the matrices $\Phi$ and $M$ in (2.30) respectively. Thus we get

$$
\begin{aligned}
\left(V_{t, \tau, s, a}-s_{0}\right) W_{t, \tau, s, a}^{2,0}= & \frac{s \Phi_{11}(t, \tau)-s_{0}}{\Phi_{11}(t, \tau)^{2}} \partial_{s s}+\frac{2\left(s_{0}-s \Phi_{11}(t, \tau)\right) \Phi_{21}(t, \tau)}{\Phi_{11}(t, \tau)^{2}} \partial_{s a} \\
& +\frac{\left(s \Phi_{11}(t, \tau)-s_{0}\right) \Phi_{21}(t, \tau)^{2}}{\Phi_{11}(t, \tau)^{2}} \partial_{a a}+\frac{M_{11}(t, \tau)}{\Phi_{11}(t, \tau)} \partial_{s s s} \\
& +\frac{M_{21}(t, \tau)-2 M_{11}(t, \tau) \Phi_{21}(t, \tau)}{\Phi_{11}(t, \tau)} \partial_{s s a} \\
& +\frac{\Phi_{21}(t, \tau)\left(M_{11}(t, \tau) \Phi_{21}(t, \tau)-2 M_{21}(t, \tau)\right)}{\Phi_{11}(t, \tau)} \partial_{s a a} \\
& +\frac{M_{21}(t, \tau) \Phi_{21}(t, \tau)^{2}}{\Phi_{11}(t, \tau)} \partial_{a a a} .
\end{aligned}
$$

Reordering all terms, we obtain the following expression for the coefficients $f_{i-j, j, k}^{1}$ in (2.45):

$$
\begin{aligned}
& f_{2,0,0}^{1}(t, T)=-s_{0} \int_{t}^{T} \frac{\alpha_{1}(\tau)}{\Phi_{11}(t, \tau)^{2}} d \tau \\
& f_{1,1,0}^{1}(t, T)=2 s_{0} \int_{t}^{T} \alpha_{1}(\tau) \frac{\Phi_{21}(t, \tau)}{\Phi_{11}(t, \tau)^{2}} d \tau, \\
& f_{0,2,0}^{1}(t, T)=-s_{0} \int_{t}^{T} \alpha_{1}(\tau) \frac{\Phi_{21}(t, \tau)^{2}}{\Phi_{11}(t, \tau)^{2}} d \tau, \\
& f_{3,0,0}^{1}(t, T)=\int_{t}^{T} \alpha_{1}(\tau) \frac{M_{11}(t, \tau)}{\Phi_{11}(t, \tau)} d \tau, \\
& f_{2,1,0}^{1}(t, T)=\int_{t}^{T} \alpha_{1}(\tau) \frac{M_{21}(t, \tau)-2 M_{11}(t, \tau) \Phi_{21}(t, \tau)}{\Phi_{11}(t, \tau)} d \tau,
\end{aligned}
$$




$$
\begin{aligned}
& f_{1,2,0}^{1}(t, T)=\int_{t}^{T} \alpha_{1}(\tau) \frac{\Phi_{21}(t, \tau)\left(M_{11}(t, \tau) \Phi_{21}(t, \tau)-2 M_{21}(t, \tau)\right)}{\Phi_{11}(t, \tau)} d \tau \\
& f_{0,3,0}^{1}(t, T)=\int_{t}^{T} \alpha_{1}(\tau) \frac{M_{21}(t, \tau) \Phi_{21}(t, \tau)^{2}}{\Phi_{11}(t, \tau)} d \tau \\
& f_{2,0,1}^{1}(t, T)=\int_{t}^{T} \frac{\alpha_{1}(\tau)}{\Phi_{11}(t, \tau)} d \tau \\
& f_{1,1,1}^{1}(t, T)=-2 \int_{t}^{T} \alpha_{1}(\tau) \frac{\Phi_{21}(t, \tau)}{\Phi_{11}(t, \tau)} d \tau \\
& f_{0,2,1}^{1}(t, T)=\int_{t}^{T} \alpha_{1}(\tau) \frac{\Phi_{21}(t, \tau)^{2}}{\Phi_{11}(t, \tau)} d \tau, \\
& f_{3,0,1}^{1}(t, T)=f_{2,1,1}^{1}(t, T)=f_{1,2,1}^{1}(t, T)=f_{0,3,1}^{1}(t, T)=0 .
\end{aligned}
$$

Having the explicit representation of $J_{t, T, s}^{1}$, from (2.40) we directly get the expression of $\hat{J}_{t, \tau, T, s, a}^{1}$ :

$$
\hat{J}_{t, \tau, T, s, a}^{1}=\sum_{k=0}^{1} \sum_{i=2}^{3} \sum_{j=0}^{i} f_{i-j, j, k}^{1}(t, T) V_{t, \tau, s, a}^{k} W_{t, \tau, s, a}^{i-j, j} .
$$

Plugging (2.46) into (2.39) with $n=2$, we can easily find $J_{t, T, s, a}^{2}$ and $\hat{J}_{t, \tau, T, s, a}^{2}$. By an analogous iterative procedure, we can compute the higher order approximation formulae. In Section 3, we present some experiment where we computed explicitly the operators $J_{t, T, s, a}^{n}$ up to the third order for $r \neq q$ and up to the fifth order for $r=q$, to get very accurate results.

\subsection{Time-independent coefficients}

As an illustrative example, we work out the approximation formulae for the density and the fixed-strike arithmetic Asian Call in a local volatility model with time-independent coefficients: the BS and the Constant Elasticity of Variance (CEV) models are meaningful particular cases. Hence we assume the following risk-neutral dynamics for the asset

$$
d S_{t}=(r-q) S_{t} d t+\sigma\left(S_{t}\right) S_{t} d W_{t} .
$$

We set

$$
\alpha(s)=\sigma^{2}(s) s^{2}, \quad \mu=r-q,
$$

and consider the pricing operator

$$
L=\frac{\alpha(s)}{2} \partial_{s s}+\mu s \partial_{s}+s \partial_{a}+\partial_{t}
$$

We also fix $s_{0}>0$ and put

$$
\alpha_{0}=\alpha\left(s_{0}\right), \quad \alpha_{k}=\frac{1}{2 k !} \partial_{s}^{k} \alpha\left(s_{0}\right), \quad k \geq 1 .
$$


Then

$$
L^{0}=\frac{\alpha_{0}}{2} \partial_{s s}+\mu s \partial_{s}+s \partial_{a}+\partial_{t}
$$

is the leading term in the approximation of $L$. Since the parameters are time independent, it is not restrictive to assume $t=0$ : accordingly, we simplify the notations and we write $\Gamma(s, a ; T, S, A)$ and $J_{T, s, a}^{1}$ instead of $\Gamma(0, s, a ; T, S, A)$ and $J_{0, T, s, a}^{1}$ respectively. The fundamental solution of $L^{0}$ is given by

$$
\Gamma^{0}(s, a ; T, S, A)=\frac{1}{2 \pi \sqrt{\operatorname{det} C(T)}} e^{-\frac{1}{2}\left\langle C^{-1}(T)\left((S, A)-m_{s, a}(T)\right),(S, A)-m_{s, a}(T)\right\rangle},
$$

with $m_{s, a}(T) \equiv m_{0, s, a}(T)$ as in (2.31) and $C(T) \equiv C(0, T)$ as in (2.20)(2.31).

For simplicity, we assume $\mu \neq 0$ and report only the first order formulae: the Mathematica notebook of higher order approximations is available in the web-site of the authors.

By Theorem 2.7 the 1-st order approximation for the density is given by

$$
\Gamma^{1}(s, a ; T, S, A)=\Gamma^{0}(s, a ; T, S, A)+J_{T, s, a}^{1} \Gamma^{0}(s, a ; T, S, A)
$$

where

$$
J_{T, s, a}^{1}=\sum_{k=0}^{1} s^{k} \sum_{i=2}^{3} \sum_{j=0}^{i} f_{i-j, j, k}^{1}(T) \frac{\partial^{i}}{\partial s^{i-j} \partial a^{j}},
$$

and $f_{i-j, j, k}^{1}(T) \equiv f_{i-j, j, k}^{1}(0, T)$ are the deterministic functions defined in Remark 2.8: specifically, in the case of time-independent coefficients, we have

$$
f_{i-j, j, k}^{1}(T)=\alpha_{1} \alpha_{i-3} g_{i-j, j, k}(T)
$$

with $\alpha_{-1}=1, \alpha_{i}$ as in (2.48) for $i=0,1$ and where

$$
\begin{aligned}
& g_{2,0,0}=\frac{\left(e^{-2 T \mu}-1\right) s_{0}}{2 \mu}, \quad g_{1,1,0}=\frac{e^{-2 T \mu}\left(e^{T \mu}-1\right)^{2} s_{0}}{\mu^{2}}, \\
& g_{0,2,0}=\frac{s_{0}\left(3+e^{-2 T \mu}-4 e^{-T \mu}-2 T \mu\right)}{2 \mu^{3}}, \quad g_{2,0,1}=\frac{\left(1-e^{-T \mu}\right)}{\mu}, \\
& g_{1,1,1}=-\frac{2\left(-1+e^{-T \mu}+T \mu\right)}{\mu^{2}}, \quad g_{0,2,1}=-\frac{2(T \mu-\sinh (T \mu))}{\mu^{3}}, \\
& g_{3,0,0}=\frac{\left(2+e^{-3 T \mu}-3 e^{-T \mu}\right)}{6 \mu^{2}}, \\
& g_{2,1,0}=\frac{\left(1-T \mu+e^{-2 T \mu}(-1-\sinh (T \mu))\right.}{\mu^{3}}, \\
& g_{1,2,0}=\frac{e^{-3 T \mu}\left(1-e^{T \mu}\right)^{4}}{2 \mu^{4}}, \\
& g_{0,3,0}=-\frac{\left(-e^{-3 T \mu}+6 e^{-2 T \mu}-18 e^{-T \mu}+3 e^{T \mu}+2(5-6 T \mu)\right)}{6 \mu^{5}},
\end{aligned}
$$


and $g_{i-j, j, k}=0$ when $i+k=4$. Notice that the functions in (2.51) are model independent: the particular form of the volatility enters in the approximation formula only through $\Gamma^{0}$ and the coefficients $\alpha_{n}$ of the Taylor expansion of the volatility function.

Accordingly, the first order approximation for the fixed-strike Asian Call is given by

$$
e^{-r T} u(s, a, T),
$$

where

$$
u(s, a, T)=C_{\mathrm{BS}}(s, a, T)+\sum_{k=0}^{1} s^{k} \sum_{i=2}^{3} \sum_{j=0}^{i} f_{i-j, j, k}^{1}(T) \frac{\partial^{i}}{\partial s^{i-j} \partial a^{j}} C_{\mathrm{BS}}(s, a, T),
$$

and

$$
\begin{aligned}
C_{\mathrm{BS}}(s, a, T)= & \int_{\mathbb{R}} \int_{\mathbb{R}} \Gamma^{0}(s, a ; T, S, A)\left(\frac{A}{T}-K\right)^{+} d S d A \\
= & \frac{e^{-\frac{\mu \beta(s, a, T)^{2}}{\gamma(T)}} \sqrt{\mu^{3} \gamma(T)}}{2 T \mu^{3} \sqrt{\pi}} \\
& -\frac{\mu^{2} \beta(s, a, T)}{T \mu^{3}}\left(1-\mathcal{N}\left(\beta(s, a, T) \sqrt{\frac{2 \mu}{\gamma(T)}}\right)\right)
\end{aligned}
$$

with

$$
\beta(s, a, T)=s-s e^{T \mu}-a \mu+K T \mu, \quad \gamma(T)=\alpha_{0}\left(3-4 e^{\mu T}+e^{2 \mu T}+2 \mu T\right) .
$$

In the above formula, numerical errors due to cancellations for short maturities can be corrected by using the resulting series expansion. We also remark that a suitable choice of $s_{0}$ may improve the accuracy of the approximation formula: as we shall see in Section 3, in most cases $s_{0}=s$ is a convenient choice that allows to get very accurate results.

\section{$3 \quad$ Numerical experiments}

In this section our approximation formulae are tested and compared with method proposed by Linetsky [34], the Mellin transform based method (Mellin500) of Shaw [39], the PDE method of Vecer [45], the matched asymptotic expansions of Dewynne and Shaw [12] (MAE3 and MAE5) and the method of Dassios and Nagaradjasarma [11] (DN). Our 2nd, 3rd and 5th order approximations will be denoted by FPP2, FPP3 and FPP5. In the first part of this section a set of experimental results under BS dynamics are reported, then in the second part the CEV dynamics is considered. 


\subsection{Tests under Black \& Scholes dynamics}

In order to assess the performances of our approximations for pricing arithmetic Call options under a BS model, we used the family of tests introduced in [20], and later used in [11, 12, 15, 19, 34, 45] as a standard for this task. Table 1 reports the interest rate $r$, the volatility $\sigma$, the time to maturity $T$, the strike $K$ and the initial asset price $S_{0}$ for the seven cases. In this set of tests a null dividend rate is assumed: $q=0$. Table 2 reports

\begin{tabular}{c|lllll}
\hline \hline Case & $S_{0}$ & $K$ & $r$ & $\sigma$ & $T$ \\
\hline 1 & 2 & 2 & 0.02 & 0.1 & 1 \\
2 & 2 & 2 & 0.18 & 0.3 & 1 \\
3 & 2 & 2 & 0.0125 & 0.25 & 2 \\
4 & 1.9 & 2 & 0.05 & 0.5 & 1 \\
5 & 2 & 2 & 0.05 & 0.5 & 1 \\
6 & 2.1 & 2 & 0.05 & 0.5 & 1 \\
7 & 2 & 2 & 0.05 & 0.5 & 2 \\
\hline \hline
\end{tabular}

Table 1: Parameter values for seven test cases

\begin{tabular}{c|cccccc}
\hline \hline Case & Linetsky & FPP3 & FPP2 & Mellin500 & Vecer & MAE3 \\
\hline 1 & 0.05598604 & 0.05598604 & 0.05598602 & 0.05603631 & 0.055986 & 0.05598596 \\
2 & 0.21838755 & 0.21838706 & 0.21838375 & 0.21835987 & 0.218388 & 0.21836866 \\
3 & 0.17226874 & 0.17226694 & 0.17226600 & 0.17236881 & 0.172269 & 0.17226265 \\
4 & 0.19317379 & 0.19316359 & 0.19320627 & 0.19297162 & 0.193174 & 0.19318824 \\
5 & 0.24641569 & 0.24640562 & 0.24640056 & 0.24651870 & 0.246416 & 0.24638175 \\
6 & 0.30622036 & 0.30620974 & 0.30615763 & 0.30649701 & 0.306220 & 0.30613888 \\
7 & 0.35009522 & 0.35003972 & 0.35001419 & 0.34892612 & 0.350095 & 0.34990862 \\
\hline \hline
\end{tabular}

Table 2: Asian Call Option Prices when $q=0$ (parameters as in Table 1)

the results of methods Linetsky, FPP3, FPP2, Mellin500, Vecer and MAE3. The results of Linetsky, Vecer and MAE3 are taken from [34], [45] and [12], respectively.

Following [12] we repeated the same seven tests with a dividend rate equal to the interest rate (see Table 3). The results of Linetsky and Vecer are not reported: the former because these tests were not considered in his paper; the latter because Vecer's code cannot deal with that special case. In that case, the discrepancies between FPP3 and MAE5 can be found only at the 5th decimal place. Furthermore, FPP5 and MAE5 columns show that the contribution of the 5 th order approximations to the accuracy of the methods is not substantial.

Next, in order to address the issues raised in Shaw [38], Fu, Madan and Wang [19] and Dufresne [17], we tested our method with a low-volatility parameter $\sigma=0.01$. Table 4 shows the performances of the approximations against Monte Carlo 95\% confidence intervals. These intervals are computed using 500000 Monte Carlo replication and an Euler discretization with 300 


\begin{tabular}{c|ccc|cc}
\hline \hline Case & FPP5 & FPP3 & FPP2 & MAE3 & MAE5 \\
\hline 1 & 0.045143 & 0.045143 & 0.045143 & 0.045143 & 0.045143 \\
2 & 0.115188 & 0.115188 & 0.115188 & 0.011518 & 0.115188 \\
3 & 0.158380 & 0.158378 & 0.158378 & 0.158378 & 0.158380 \\
4 & 0.169201 & 0.169192 & 0.169238 & 0.169238 & 0.169201 \\
5 & 0.217815 & 0.217805 & 0.217805 & 0.217805 & 0.217815 \\
6 & 0.272924 & 0.272914 & 0.272868 & 0.272869 & 0.272925 \\
7 & 0.291316 & 0.291263 & 0.291263 & 0.291264 & 0.291316 \\
\hline \hline
\end{tabular}

Table 3: Asian Call Option Prices when $q=r$ (parameters as in Table 1).

time-steps for $T=0.25$ and $T=1$ and 1500 time-steps for $T=5$. In these experiments the initial asset level is $S_{0}=100$, the interest rate is $r=0.05$ and the dividend yield is null $q=0$.

The methods considered are Vecer, Mellin500, FPP3 and MAE3. Here, we used the Mathematica implementations of Vecer and Mellin500 provided by the authors, whereas MAE3 formula was coded by ourself. Mellin500 implementation requires a numerical integration on an unbounded domain which needs to be truncated. We have set the length of the truncated domain to $10^{9}$ and fixed the number of recursion in Mathematica NIntegrate function to 100. The execution time of the Monte Carlo, Vecer and Mellin500 methods is also reported. Also here, FPP3 and MAE3 methods are almost identical and both always fall very close to Monte Carlo results: the worst case has an error of $5 \times 10^{-3}$. Notice that the Euler discretization may induce a little bias in Monte Carlo results.

We remark that, although the proposed approximations have a performance very similar to the method of Dewynne and Shaw, our approach is more flexible and capable of dealing with local volatility dynamics; moreover, our method can also produce explicit approximation formulae for the Greeks and the asset-average density. 


\begin{tabular}{|c|c|c|c|c|c|c|c|c|c|c|}
\hline \multirow[t]{2}{*}{$T$} & \multirow[t]{2}{*}{$\bar{K}$} & \multicolumn{3}{|c|}{ Euler - Monte Carlo method } & \multicolumn{2}{|l|}{ Vecer } & \multicolumn{2}{|c|}{ Mellin500 } & \multirow[t]{2}{*}{ FPP3 } & \multirow[t]{2}{*}{ MAE3 } \\
\hline & & \multicolumn{2}{|c|}{$95 \%$ c.i. } & ex. time & value & ex. time & value & ex. time & & \\
\hline 0.25 & 99 & $1.60849 \times 10^{0}$ & $1.61008 \times 10^{0}$ & 70.52 & $-4.18937 \times 10^{1}$ & 0.022 & $1.51718 \times 10^{0}$ & 4.09 & $1.60739 \times 10^{0}$ & $1.60739 \times 10^{0}$ \\
\hline 0.25 & 100 & $6.22333 \times 10^{-1}$ & $6.23908 \times 10^{-1}$ & 70.64 & $5.40466 \times 10^{-1}$ & 0.022 & $6.96855 \times 10^{-1}$ & 4.08 & $6.21359 \times 10^{-1}$ & $6.21359 \times 10^{-1}$ \\
\hline 0.25 & 101 & $1.39301 \times 10^{-2}$ & $1.42436 \times 10^{-2}$ & 71.02 & $-3.96014 \times 10^{-2}$ & 0.022 & $1.60361 \times 10^{-1}$ & 4.09 & $1.37618 \times 10^{-2}$ & $1.37615 \times 10^{-2}$ \\
\hline 1.00 & 97 & $5.27670 \times 10^{0}$ & $5.27985 \times 10^{0}$ & 70.91 & $-9.73504 \times 10^{0}$ & 0.019 & $5.27474 \times 10^{0}$ & 4.38 & $5.27190 \times 10^{0}$ & $5.27190 \times 10^{0}$ \\
\hline 1.00 & 100 & $2.42451 \times 10^{0}$ & $2.42767 \times 10^{0}$ & 70.89 & $2.37512 \times 10^{0}$ & 0.020 & $2.43303 \times 10^{0}$ & 4.26 & $2.41821 \times 10^{0}$ & $2.41821 \times 10^{0}$ \\
\hline 1.00 & 103 & $7.44026 \times 10^{-2}$ & $7.54593 \times 10^{-2}$ & 70.61 & $7.25478 \times 10^{-2}$ & 0.020 & $8.50816 \times 10^{-2}$ & 4.24 & $7.26910 \times 10^{-2}$ & $7.24337 \times 10^{-2}$ \\
\hline 5.00 & 80 & $2.61775 \times 10^{1}$ & $2.61840 \times 10^{1}$ & 316.62 & $2.52779 \times 10^{1}$ & 0.018 & $2.61756 \times 10^{1}$ & 4.40 & $2.61756 \times 10^{1}$ & $2.61756 \times 10^{1}$ \\
\hline 5.00 & 120 & $1.41956 \times 10^{-6}$ & $1.38366 \times 10^{-5}$ & 284.00 & $1.07085 \times 10^{-5}$ & 0.017 & $1.42235 \times 10^{-3}$ & 2.62 & $2.06699 \times 10^{-5}$ & $5.73317 \times 10^{-6}$ \\
\hline
\end{tabular}

Table 4: Tests with low volatility: $\sigma=0.01, S_{0}=100, r=0.05$ and $q=0$ 


\subsection{Tests under CEV dynamics}

In this section we test the performances of our approximation when the volatility is not constant. More specifically, we consider the CEV dynamics

$$
\left.d S_{t}=(r-q) d S_{t} d t+\sigma S^{\beta} d W_{t}, \quad \beta \in\right] 0,1[,
$$

which corresponds to a local volatility model with $\sigma(t, S)=\sigma S^{\beta-1}$. Although this is a "degenerate" case, as $\sigma(t, S)$ is not bounded, the following experiments confirm that the approximation is still precise enough.

Firstly, we performed the experiments proposed by Dassios and Nagaradjasarma in [11] for the square-root model, $\beta=\frac{1}{2}$. The results on these tests are reported in Tables 5 and 6 , where the 2-nd and 3-rd order approximations are compared with the results of a Monte Carlo method. The same number of Monte Carlo replications and time-steps of previous experiments was used. Here again, both FPP2 and FPP3 approximations show good performances.

\begin{tabular}{c|ccccc|ccc|c}
\hline \hline Case & $S_{0}$ & $K$ & $r$ & $\sigma$ & $T$ & DN & FPP3 & FPP2 & MC 95\% c.i. \\
\hline $1^{\star}$ & 2 & 2 & 0.02 & 0.14 & 1 & 0.0197 & 0.055562 & 0.055562 & $0.055321-0.055732$ \\
$2^{\star}$ & 2 & 2 & 0.18 & 0.42 & 1 & 0.2189 & 0.217874 & 0.217875 & $0.218319-0.219678$ \\
$3^{\star}$ & 2 & 2 & 0.0125 & 0.35 & 2 & 0.1725 & 0.170926 & 0.170926 & $0.171126-0.172555$ \\
$4^{\star}$ & 1.9 & 2 & 0.05 & 0.69 & 1 & 0.1902 & 0.190834 & 0.190821 & $0.190303-0.192121$ \\
$5^{\star}$ & 2 & 2 & 0.05 & 0.72 & 1 & NA & 0.251121 & 0.251123 & $0.250675-0.252807$ \\
$6^{\star}$ & 2.1 & 2 & 0.05 & 0.72 & 1 & 0.3098 & 0.308715 & 0.308730 & $0.308791-0.311150$ \\
$7^{\star}$ & 2 & 2 & 0.05 & 0.71 & 2 & 0.3339 & 0.353197 & 0.353206 & $0.352269-0.355313$ \\
\hline \hline
\end{tabular}

Table 5: Tests proposed by Dassios and Nagardjasarma [11] for the CEV model.

\begin{tabular}{cc|ccc|c}
\hline \hline$\sigma$ & $T$ & DN & FPP3 & FPP2 & MC 95\% c.i. \\
\hline 0.71 & 0.1 & 0.0751 & 0.075387 & 0.075387 & $0.075068-0.075689$ \\
0.71 & 0.5 & 0.1725 & 0.173175 & 0.173175 & $0.173265-0.174717$ \\
0.71 & 1.0 & 0.2468 & 0.248018 & 0.248019 & $0.247738-0.249841$ \\
0.71 & 2.0 & 0.3339 & 0.353197 & 0.353206 & $0.351111-0.354146$ \\
0.71 & 5.0 & 0.3733 & 0.545714 & 0.545800 & $0.545812-0.550679$ \\
0.1 & 1 & 0.0484 & 0.061439 & 0.061439 & $0.061329-0.061674$ \\
0.3 & 1 & 0.1207 & 0.120680 & 0.120680 & $0.120596-0.121494$ \\
0.5 & 1 & 0.1827 & 0.182723 & 0.182724 & $0.182814-0.184285$ \\
0.7 & 1 & 0.2446 & 0.244913 & 0.244914 & $0.244959-0.247030$ \\
\hline \hline
\end{tabular}

Table 6: Second set of tests proposed by Dassios and Nagardjasarma [11]. The remaining parameters are set to $S_{0}=K=2, r=0.05, q=0$ and $\beta=\frac{1}{2}$

Figure 1 and 2 show the cross-sections of absolute (left) and relative (right) errors of the 3 -rd order approximation when $\beta=\frac{2}{3}$ and $\beta=\frac{1}{3}$, respectively. The errors are computed against prices computed by means of an Euler Monte Carlo method with 300 time-steps and 500000 replications. 
The shaded bands show the $95 \%$ and $99 \%$ Monte Carlo confidence intervals for each strike. The initial stock price is $S_{0}=1$, the risk-free rate is $r=5 \%$, the dividend yield is $q=0$ and the maturity is $T=1$. Two levels for the volatility parameter are considered $\sigma=10 \%$ and $\sigma=50 \%$. The two figures show that the approximations have good global performances for both the CEV exponents and both the volatility levels.

Finally, since the approach here proposed is capable of approximating analytically also the sensitivities of option prices, we show in Figure 3 the graphs of the Delta, the Gamma and the Vega of an arithmetic Asian Call, with fixed strike, under the CEV model. Notice that, usual no-arbitrage bounds, like having the Delta in the interval $[0,1]$ or positive Gamma, are not violated by the approximation formulae.

\section{References}

[1] A. A. Agrachev and Y. L. Sachkov, Control theory from the geometric viewpoint, vol. 87 of Encyclopaedia of Mathematical Sciences, Springer-Verlag, Berlin, 2004. Control Theory and Optimization, II.

[2] J. E. Angus, A note on pricing Asian derivatives with continuous geometric averaging, Journal of Futures Markets, 19 (1999), pp. 845858.

[3] V. Bally and A. Kohatsu-Higa, Lower bounds for densities of Asian type stochastic differential equations, J. Funct. Anal., 258 (2010), pp. 3134-3164.

[4] E. Barucci, S. Polidoro, And V. Vespri, Some results on partial differential equations and Asian options, Math. Models Methods Appl. Sci., 11 (2001), pp. 475-497.

[5] E. Bayraktar and H. XIng, Pricing Asian options for jump diffusion, Math. Finance, 21 (2011), pp. 117-143.

[6] P. Boyle, M. Broadie, and P. Glasserman, Monte Carlo methods for security pricing, J. Econom. Dynam. Control, 21 (1997), pp. 12671321. Computational financial modelling.

[7] N. Cai And S. G. Kou, Pricing Asian options under a HyperExponential jump diffusion model, to appear in Operations Research, (2011).

[8] N. C. Caister, J. G. O'Hara, and K. S. Govinder, Solving the Asian option PDE using Lie symmetry methods, Int. J. Theor. Appl. Finance, 13 (2010), pp. 1265-1277. 

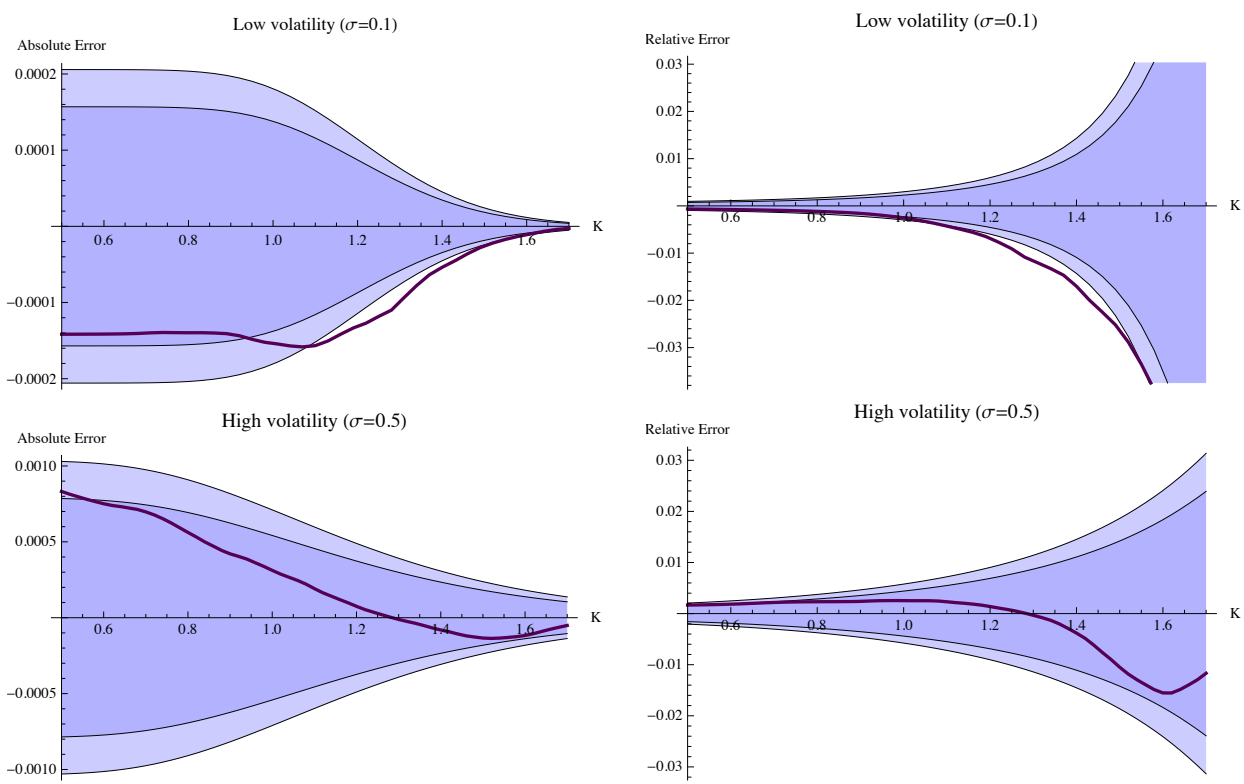

Figure 1: FPP3 approximation errors for Asian Call options with maturity $T=1$, when the underlying has a CEV dynamics with exponent $\beta=\frac{2}{3}$ and parameters $S_{0}=1, r=5 \%$ and $q=0$. Two levels of volatility are considered: $\sigma=10 \%$ and $\sigma=50 \%$
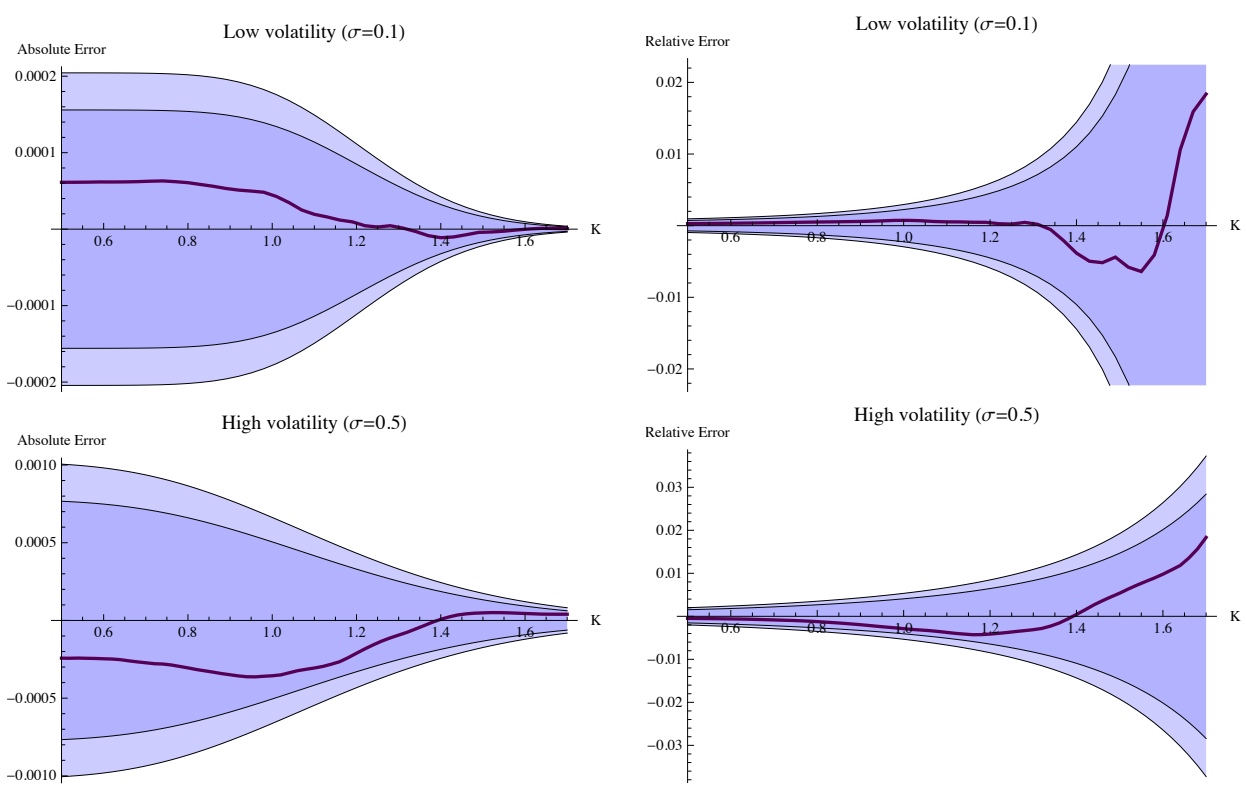

Figure 2: FPP3 approximation errors for Asian Call options with maturity $T=1$, when the underlying has a CEV dynamics with exponent $\beta=\frac{1}{3}$ and parameters $S_{0}=1, r=5 \%$ and $q=0$. Two levels of volatility are considered: $\sigma=10 \%$ and $\sigma=50 \%$ 

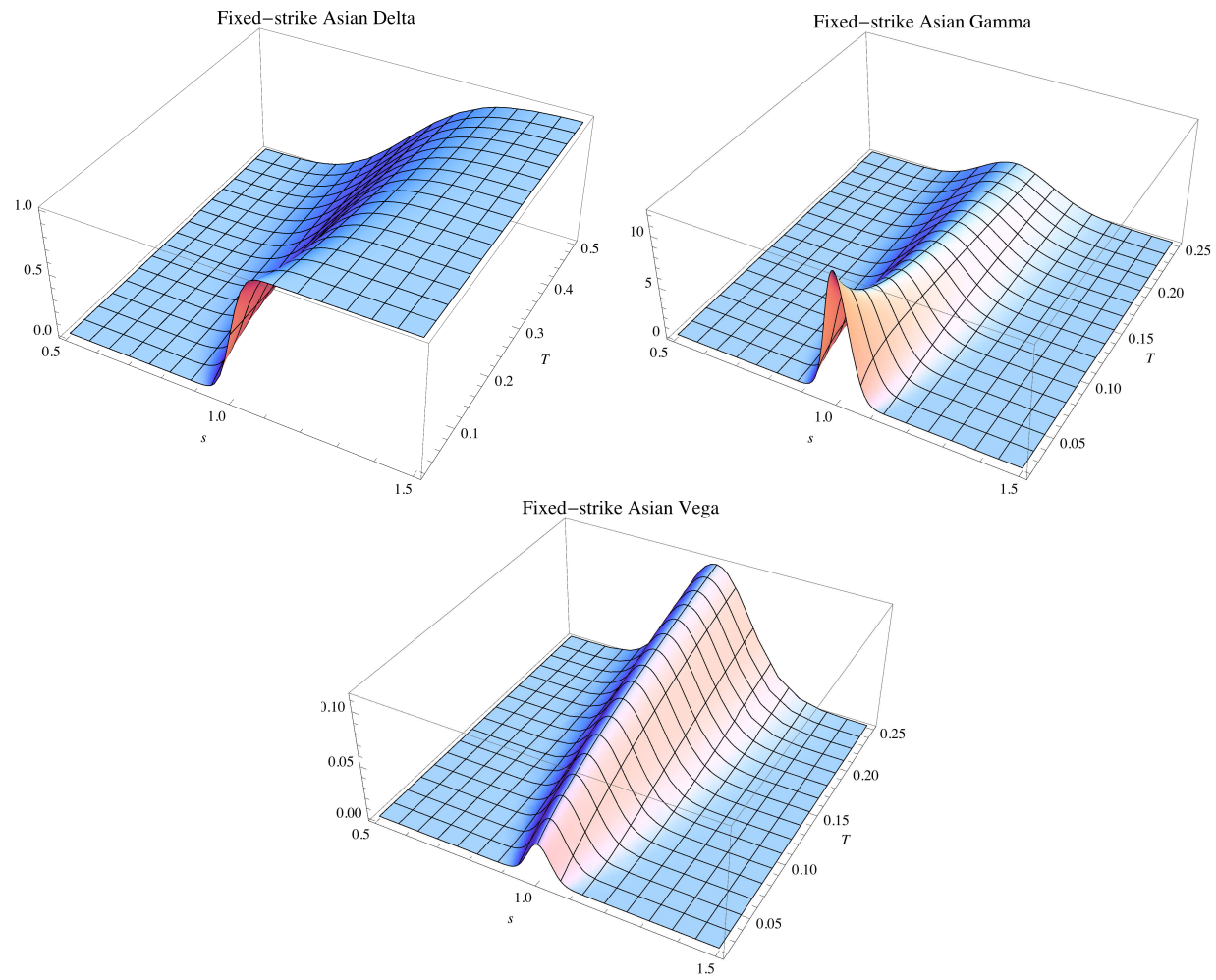

Figure 3: Delta, Gamma and Vega of an arithmetic Average Call with fixed strike $K=1$, under a CEV dynamics with parameters $r=5 \%, q=0$, $\sigma=40 \%$ and $\beta=\frac{1}{2}$

[9] F. Corielli, P. Foschi, And A. Pascucci, Parametrix approximation of diffusion transition densities, SIAM J. Financial Math., 1 (2010), pp. 833-867.

[10] J.-M. Coron, Control and nonlinearity, vol. 136 of Mathematical Surveys and Monographs, American Mathematical Society, Providence, RI, 2007.

[11] A. Dassios And J. Nagaradjasarma, The square-root process and Asian options, Quant. Finance, 6 (2006), pp. 337-347.

[12] J. N. Dewynne And W. T. Shaw, Differential equations and asymptotic solutions for arithmetic Asian options: 'Black-Scholes formulae' for Asian rate calls, European J. Appl. Math., 19 (2008), pp. 353-391.

[13] J. N. Dewynne And P. Wilmott, A note on average rate options with discrete sampling, SIAM J. Appl. Math., 55 (1995), pp. 267-276. 
[14] M. Di Francesco, A. Pascucci, and S. Polidoro, The obstacle problem for a class of hypoelliptic ultraparabolic equations, Proc. R. Soc. Lond. Ser. A Math. Phys. Eng. Sci., 464 (2008), pp. 155-176.

[15] D. Dufresne, Laguerre series for Asian and other options, Math. Finance, 10 (2000), pp. 407-428.

[16] — The integrated square-root process, (2001).

[17] — Asian and Basket asymptotics, Research paper n.100, University of Montreal, (2002).

[18] P. Foschi and A. Pascucci, Path dependent volatility, Decis. Econ. Finance, 31 (2008), pp. 13-32.

[19] M. Fu, D. Madan, And T. Wang, Pricing continuous time Asian options: a comparison of Monte Carlo and Laplace transform inversion methods, J. Comput. Finance, 2 (1998), pp. 49-74.

[20] H. Geman and A. Eydeland, Domino effect, RISK, 8 (1995), pp. 6567.

[21] H. Geman and M. Yor, Quelques relations entre processus de Bessel, options asiatiques et fonctions confluentes hypergéométriques, C. R. Acad. Sci. Paris Sér. I Math., 314 (1992), pp. 471-474.

[22] S. Glasgow and S. Taylor, A novel reduction of the simple Asian option and Lie-group invariant solutions, Int. J. Theor. Appl. Finance, 12 (2009), pp. 1197-1212.

[23] E. Gobet, Approximations of stochastic differential equations: an overview, 7th Seminar on Stochastic Analysis, Random Fields and Applications - Ascona, (23-27 May 2011).

[24] P. Guasoni and S. Robertson, Optimal importance sampling with explicit formulas in continuous time, Finance Stoch., 12 (2008), pp. 119.

[25] V. Henderson and R. Wojakowski, On the equivalence of floatingand fixed-strike Asian options, J. Appl. Probab., 39 (2002), pp. 391394.

[26] D. G. Hobson And L. C. G. Rogers, Complete models with stochastic volatility, Math. Finance, 8 (1998), pp. 27-48.

[27] L. Hörmander, Hypoelliptic second order differential equations, Acta Math., 119 (1967), pp. 147-171. 
[28] J. E. Ingersoll, Theory of Financial Decision Making, Blackwell, Oxford, 1987.

[29] B. Jourdain And M. Sbai, Exact retrospective Monte Carlo computation of arithmetic average Asian options, Monte Carlo Methods Appl., 13 (2007), pp. 135-171.

[30] R. E. Kalman, Y. C. Ho, and K. S. Narendra, Controllability of linear dynamical systems, Contributions to Differential Equations, 1 (1963), pp. 189-213.

[31] A. G. Z. Kemna And A. C. F. Vorst, A pricing method for options based on average asset values, Journal of Banking and Finance, 14 (1990), pp. 113-129.

[32] N. Kunitomo and A. Takahashi, Pricing average options, Japan Financial Review, 14 (1992), pp. 1-20 (in Japanese).

[33] J. P. LaSalle, The time optimal control problem, in Contributions to the theory of nonlinear oscillations, Vol. V, Princeton Univ. Press, Princeton, N.J., 1960, pp. 1-24.

[34] V. Linetsky, Spectral expansions for Asian (average price) options, Oper. Res., 52 (2004), pp. 856-867.

[35] A. PASCUCCI, Free boundary and optimal stopping problems for American Asian options, Finance Stoch., 12 (2008), pp. 21-41.

[36] A. Pascucci and S. Pagliarani, Analytical approximation of the transition density in a local volatility model, SSRN eLibrary, (2011).

[37] L. Rogers And Z. Shi, The value of an Asian option., J. Appl. Probab., 32 (1995), pp. 1077-1088.

[38] W. T. SHAw, Modelling financial derivatives with Mathematica, Cambridge University Press, Cambridge, 1998. Mathematical models and benchmark algorithms, With 1 CD-ROM (Windows, Macintosh and UNIX).

[39] — Pricing Asian options by contour integration, including asymptotic methods for low volatility, Working paper,

http://www.mth.kcl.ac.uk/ shaww/web_page/papers/Mathematicafin.htm, (2003).

[40] K. Shiraya And A. TAKahashi, Pricing average options on commodities, forthcoming in Journal of Futures Markets, (2010). 
[41] K. Shiraya, A. Takahashi, and M. Toda, Pricing Barrier and Average Options Under Stochastic Volatility Environment, SSRN eLibrary, (2009).

[42] A. Takahashi and N. Yoshida, Monte Carlo simulation with asymptotic method, J. Japan Statist. Soc., 35 (2005), pp. 171-203.

[43] S. TAYlor, Perturbation and symmetry techniques applied to finance, Ph. D. thesis, Frankfurt School of Finance \& Management. Bankakademie HfB, (2011).

[44] J. VECER, A new pde approach for pricing arithmetic average Asian options, J. Comput. Finance, (2001), pp. 105-113.

[45] J. Vecer And M. Xu, Unified Asian pricing, Risk, 15 (2002), pp. 113116.

[46] M. Yor, On some exponential functionals of Brownian motion, Adv. in Appl. Probab., 24 (1992), pp. 509-531.

[47] _ Sur certaines fonctionnelles exponentielles du mouvement brownien réel, J. Appl. Probab., 29 (1992), pp. 202-208.

[48] J. E. ZhANG, A semi-analytical method for pricing and hedging continuously sampled arithmetic average rate options, J. Comput. Finance, 5 (2001), pp. 1-20. 